\title{
A SET WHOSE SQUARE CAN MAP ONTO A PERFECT SET
}

\author{
JOHN R. ISBELL
}

Assuming the continuum hypothesis, a set $X$ of real numbers will be constructed such that every compact continuous image of $X$ is countable, but $X \times X$ admits a uniformly continuous mapping onto the Cantor set. This completes (modulo the continuum hypothesis and the Ulam measure problem) the determination of whether a product or coproduct of $m$ Boolean algebras or fields of sets can have an infinite free, or projective, or injective sub- or quotient object when the factors do not [1]. The concluding construction (modulo the hypothesis) is embarrassingly easy, but the result is unlike the others [1]; this is the only instance of a finite coproduct creating a remarkable subobject (viz. an infinite free subfield), and there is no instance among these of a finite product creating a remarkable quotient.

The use made of the continuum hypothesis seems slight. However, Sierpiński has pointed out that the weaker result that there is a set of real numbers of the power of the continuum admitting no continuous mapping onto $[0,1]\left(C_{5}\right.$ in $\left.[2]\right)$ has not been established without the hypothesis. On the continuum hypothesis, that is a by-product of an important construction of Lusin, the subject of Chapter II of [2].

We shall find $X$ in the Cantor set $C$ considered as an infinite power of the compact group $Z_{2}$ (written additively). By a lemma of Lavrentiev (Theorem 99 of [3]), any continuous mapping of a subset of $C$ onto $[0,1]$ can be extended over a $G_{\delta}$ subset of $C$. There are only $c$ (the power of the continuum) $G_{\delta}$ sets, and each has only $c$ continuous mappings to $[0,1]$. Hence we can index all these mappings $f_{\alpha}$ by ordinals of smaller cardinal than $c$, and index similarly the points $x_{\alpha}$ of $C$. For each $f_{\alpha}$, the inverse images of points are $c$ disjoint subsets of $C$ each closed in their union; so except for countably many, they are nowhere dense. By the continuum hypothesis, $C$ is not a union of fewer than $c$ such sets.

There is no difficulty in building up sets $S, T$, no subset of either of which is mapped onto $[0,1]$ by any $f_{\alpha}$, but with every $x_{\alpha}$ representable as $s_{\alpha}+t_{\alpha}, s_{\alpha}$ in $S$ and $t_{\alpha}$ in $T$. Carry along expanding sets $H_{\alpha}$ disjoint from $S, K_{\alpha}$ disjoint from $T$ ( $H_{0}$ and $K_{0}$ empty). Arrived

Received by the editors September 29, 1967. 
at $\alpha$, add to $H_{\alpha}$ a nowhere dense inverse set of $f_{\alpha}$ containing none of the fewer than $c$ earlier points $s_{\beta}$, do likewise for $K_{\alpha}$, and find $s_{\alpha}$ in the complement of the union of $H_{\alpha}$ and the translate $x_{\alpha}+K_{\alpha}$. Let $t_{\alpha}=x_{\alpha}+s_{\alpha}\left(=x_{\alpha}-s_{\alpha}\right)$.

$S \times T$ has the indicated mapping onto $C,(s, t)$ to $s+t . S \cup T$ cannot map continuously onto $C$, since neither $S$ nor $T$ does $[1 ; 3.1]$. That proof works as well for $[0,1]$ as for $C$; but it seems worth noting that more is true. $A$ subspace of $C$ admits a continuous map onto $C$ if it admits a continuous map onto $[0,1]$. For this, cut out open-closed sets $A_{i}$ around the inverse image of the $i$ th dyadic rational $d_{i}$ and modify the map into the $\epsilon_{i}$-neighborhood of $d_{i}$ so as to omit an open neighborhood of $d_{i}$; if $2 \boldsymbol{\Sigma}_{\boldsymbol{i}}<1$, the image must be a Cantor set, and $\epsilon_{i} \rightarrow 0$ is enough to secure continuity (or uniform continuity, if one began with it). Finally, a separable metrizable space admits a continuous map onto $[0,1]$ if it has an uncountable compact (Hausdorff) continuous image. For it has no uncountable scattered image; a nondegenerate component would map to $[0,1]$ (which is injective); and a compact, dense-in-itself, totally disconnected space admits a continuous map onto $C$. Accordingly $X=S \cup T$ is the required example.

The referee observes that the italicized statements above differ little from results in some textbooks. Very likely they have been printed somewhere in at least the present generality. All remarks after the construction of $S$ and $T$ were of course just polishing.

\section{REFERENCES}

1. J. Isbell, Spaces without large projective subspaces, Math. Scand. 17 (1965), 89105; correction, ibid. 20 (1968).

2. W. Sierpinski, Hypothèse du continu, Monogr. Mat., Tom IV, Warsaw, 1934.

3. - General topology, Univ. of Toronto Press, Toronto, 1952.

Case Western Reserve University 